\title{
Enhanced graphene nonlinear response through geometrical plasmon focusing
}

J. R. M. Saavedra, and F. Javier García de Abajo

Citation: Appl. Phys. Lett. 112, 061107 (2018); doi: 10.1063/1.5017120

View online: https://doi.org/10.1063/1.5017120

View Table of Contents: http://aip.scitation.org/toc/apl/112/6

Published by the American Institute of Physics

\section{Articles you may be interested in}

Liquid crystal mediated active nano-plasmonic based on the formation of hybrid plasmonic-photonic modes Applied Physics Letters 112, 061101 (2018); 10.1063/1.5004076

Polarization control of high transmission/reflection switching by all-dielectric metasurfaces

Applied Physics Letters 112, 063103 (2018); 10.1063/1.5018783

Cavity enhanced third harmonic generation in graphene

Applied Physics Letters 112, 011102 (2018); 10.1063/1.4999054

Contact mechanics of graphene-covered metal surfaces

Applied Physics Letters 112, 061601 (2018); 10.1063/1.5006770

Probing collective oscillation of d-orbital electrons at the nanoscale

Applied Physics Letters 112, 061102 (2018); 10.1063/1.5012742

Giant excitation induced bandgap renormalization in TMDC monolayers

Applied Physics Letters 112, 061104 (2018); 10.1063/1.5017069

\section{PHYSICS TODAY}

MANAGER'S GUIDE

WHITEPAPERS

\section{READ NOW}

PRESENTED BY

Accelerate R\&D with
Multiphysics Simulation 


\title{
Enhanced graphene nonlinear response through geometrical plasmon focusing
}

\author{
J. R. M. Saavedra ${ }^{1}$ and F. Javier García de Abajo ${ }^{1,2, a)}$ \\ ${ }^{1}$ ICFO-Institut de Ciencies Fotoniques, The Barcelona Institute of Science and Technology, \\ 08860 Castelldefels (Barcelona), Spain \\ ${ }^{2}$ ICREA-Institució Catalana de Recerca i Estudis Avançats, Passeig Lluís Companys, 23, 08010 Barcelona, \\ Spain
}

(Received 24 November 2017; accepted 28 January 2018; published online 8 February 2018)

\begin{abstract}
We propose a simple approach to couple light into graphene plasmons and focus these excitations at focal spots of a size determined by the plasmon wavelength, thus producing high optical field enhancement that boosts the nonlinear response of the material. More precisely, we consider a graphene structure in which incident light is coupled to its plasmons at the carbon edges and subsequently focused on a spot of size comparable to the plasmon wavelength. We observe large confinement of graphene plasmons, materializing in small, intense focal spots, in which the extraordinary nonlinear response of this material leads to relatively intense harmonic generation. This result shows the potential of plasmon focusing in suitably edged graphene structures to produce large field confinement and nonlinear response without involving elaborated nanostructuring. Published by AIP Publishing. https://doi.org/10.1063/1.5017120
\end{abstract}

The control of light by light is a research frontier in nanophotonics, limited by the weak nonlinear response of available materials, which results in small effects within structures of sizes comparable to the light wavelength. Light concentration over small spatial regions directly produces an increase in the level of nonlinearity, and so, this approach has been extensively explored with different strategies, ranging from geometrical light concentrators to more sophisticated schemes based on coupling to ultracompact optical excitations, such as plasmons and phonon polaritons. ${ }^{1-9}$ In particular, localized plasmons can focus light on the nanometer scale, a phenomenon that is accompanied by a huge enhancement of the optical field intensity driven by coupling to evanescent fields. ${ }^{10}$ This strategy has been used to increase the intensity of nonlinear processes, ${ }^{1,3,9,11}$ although the effect is constrained by the small amount of the nonlinear material that can be allocated within the nanoplasmonic hotspots.

Direct focusing of propagating light, for example, through the use of micron-sized Fresnel's lenses, ${ }^{12}$ is limited by diffraction to regions of the order of the light wavelength $\lambda_{0}$. This situation can be improved by optical coupling to propagating modes of shorter wavelengths, such as surfaceplasmon polaritons, with a subsequent gain in field intensity. For instance, adiabatic plasmon focusing has been successfully explored in self-similar particle arrays, ${ }^{13}$ tapered ribbons, ${ }^{14,15}$ and metallic tips. ${ }^{16}$ Plasmon concentrators have also been investigated. ${ }^{17}$ An interesting theoretical study is presented in Ref. 18, where light is coupled to graphene plasmons through vacancies drawn along the carbon layer, with application in the creation of high-orbital-angular-momentum states and focusing of vortex plasmons at the center of Bessel plasmon beams. Here, we aim at producing a high concentration of two-dimensional (2D) plasmon fields and exploring its use to increase nonlinear interactions, such as harmonic

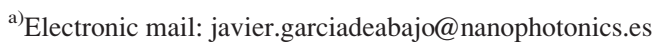

generation. In particular, we are interested in coupling to plasmons sustained by ultrathin layers of materials such as graphene $^{19-24}$ and black-phosphorous, ${ }^{25,26}$ which can sustain infrared plasmons with wavelengths $\lambda_{\mathrm{p}}$ down to a few nanometers. Additionally, plasmons in ultrathin noble metals ${ }^{27}$ can reach the visible regime with $\lambda_{\mathrm{p}}<100 \mathrm{~nm}$. This compression in the plasmon wavelength compared with the light wavelength in ultrathin films can be explained in the following way: a thin film of surface conductivity $\sigma(\omega)$ (under the condition that $\operatorname{Im}\{\sigma\}>0$, that is, it has metallic optical behavior) can sustain plasmons of wavelength $\lambda_{\mathrm{p}}=-4 \pi^{2} \mathrm{i} \sigma / \epsilon \omega$ at frequency $\omega,{ }^{23}$ where $\epsilon$ is the average permittivity of the media above and below the film; in the Drude model, a conductive film of thickness $t$, volumetric charge-carrier density $n_{\mathrm{V}}$, and carrier effective mass $m^{*}$ has $\sigma=\left(\mathrm{i} e^{2} / m^{*}\right) n_{\mathrm{V}} t / \omega$, and so, the plasmon wavelength reduces to $\lambda_{\mathrm{p}}=\left(4 \pi^{2} e^{2} / \epsilon m^{*}\right) n_{\mathrm{V}} t / \omega^{2}$; for atomic (111) monolayers of gold or silver $\left(m^{*}=1, n_{\mathrm{V}}=4 / a^{3}\right.$, and $t=a / \sqrt{3}$, where $a \approx 0.41 \mathrm{~nm}$ is the atomic lattice constant) supported on silica $(\epsilon \approx 3 / 2)$, this expression leads to $\lambda_{\mathrm{p}} / \mathrm{nm}=40\left(E_{\mathrm{p}} / \mathrm{eV}\right)^{-2}$, where $E_{\mathrm{p}}=\hbar \omega$ is the plasmon energy; for silica-supported doped graphene $\left(m^{*}=\sqrt{\pi n} \hbar / v_{\mathrm{F}}\right.$, where $n=n_{\mathrm{V}} t$ is the areal doping density and $v_{\mathrm{F}} \approx 10^{6} \mathrm{~m} / \mathrm{s}$ is the Fermi velocity), we have $\lambda_{\mathrm{p}} / \mathrm{nm}=4.4 \sqrt{n /\left(10^{13} \mathrm{~cm}^{-2}\right)}\left(E_{\mathrm{p}} / \mathrm{eV}\right)^{-2}$, subject to the condition $E_{\mathrm{p}}<\hbar v_{\mathrm{F}} \sqrt{\pi n}$ (i.e., the plasmon energy needs to be below the Fermi level to prevent interband damping). Additionally, the principle of focusing through coupling light to short- $\lambda_{\mathrm{p}}$ surface modes can be directly extended to $2 \mathrm{D}$ surface polaritons, which exist in many forms over a wide range of material platforms (e.g., van der Waals atomic layers ${ }^{28}$ ) featuring small $\lambda_{\mathrm{p}} / \lambda_{0}$ ratios.

However, a strong reduction in $\lambda_{\mathrm{p}}$ relative to $\lambda_{0}$ also implies a poor light-plasmon coupling efficiency. Despite the numerous solutions that have been explored to overcome this problem (e.g., using gratings ${ }^{29,30}$ or tips ${ }^{31}$ to increase the 
coupling), it remains as a pending challenge that reduces the applicability of plasmon focusing for enhanced nonlinear interactions. A solution to this problem is important in many practical scenarios, such as the miniaturization of nonlinear optical elements for integrated devices ${ }^{6,8}$ or to improve the signal-to-noise ratio in surface-enhanced Raman scattering $(\text { SERS) })^{32-35}$ and infrared absorption (SEIRA) ${ }^{36,37}$ for optical sensing.

In this letter, we propose a simple structure to couple incident light into plasmons and focus them at a spot of size $\sim \lambda_{\mathrm{p}}$. Specifically, we consider the structure presented in Fig. 1(a), which consists of a 2D plasmon-supporting ribbon terminated in a semicircular edge of large radius $a$ compared with the plasmon wavelength $\lambda_{\mathrm{p}}$. Because plasmons are surface-confined modes, as we noted above, external light cannot couple to them in an extended 2D film due to energymomentum mismatch. However, lateral edges break this mismatch and produce light coupling to propagating plasmons. For a semicircular geometry, these plasmons focus at the center, thus generating a hotspot [Fig. 1(b)]. Edgecoupling to plasmons is, however, a general concept: the calculations that follow can be easily adapted to any 2Dpolariton-supporting material and applied to phenomena that require a strong field enhancement; we actually discuss results for circular and elliptical structures as well. For concreteness, we focus on nonlinear harmonic generation from graphene. Besides sustaining long-lived infrared plasmons, ${ }^{24}$ this material has been observed to display a large nonlinear optical response, ${ }^{11,38,39}$ which has been argued to be increased by the effect of field amplification when the light is tuned to its plasmons. ${ }^{40-47}$

To describe the plasmon field, we assimilate the graphene edge to a distribution of in-plane source dipoles (line dipole density $\mathcal{P}_{\mathbf{s}}$ as a function of edge position $\mathbf{s}$ ) oriented along the direction of the local in-plane edge normal $\hat{\mathbf{n}}_{\mathrm{s}}$. Noting the strong confinement of the graphene plasmons, ${ }^{48}$ it is safe to neglect retardation, and so, the field produced by a single in-plane dipole $\mathbf{p}$ placed at the origin reduces to

$$
\mathbf{E}^{(1)}(\mathbf{r}, \omega)=\frac{\mathrm{i}}{2 \pi} \int \frac{d^{2} \mathbf{Q}}{Q}(\mathrm{i} \mathbf{Q}-Q \hat{\mathbf{z}})\left(1-r_{\mathrm{p}}\right) \mathbf{Q} \cdot \mathbf{p} \mathrm{e}^{\mathrm{i} \mathbf{Q} \cdot \mathbf{R}-Q z},
$$

where $\mathbf{R}=(x, y)$ are coordinates within the graphene plane $(z=0)$ and $r_{\mathrm{p}}$ is the Fresnel reflection coefficient for $\mathrm{p}$ polarization. ${ }^{48}$ For a thin film of $2 \mathrm{D}$ conductivity $\sigma(\omega)$, adopting the plasmon-pole approximation, we have $r_{\mathrm{p}} \approx Q /(Q-$ $Q_{\mathrm{p}}$ ), where $Q_{\mathrm{p}}=\mathrm{i} \epsilon \omega / 2 \pi \sigma(\omega)$ is the complex plasmon wavenumber. Now, the plasmon field produced by a graphene edge is obtained as the integral of Eq. (1) over its contour

$$
\mathbf{E}^{(1)}(\mathbf{r}, \omega)=\frac{Q_{\mathrm{p}}}{2 \pi} \oint d \mathbf{s} \int \frac{d^{2} \mathbf{Q}}{Q} \frac{\mathbf{Q}+\mathrm{i} Q \hat{\mathbf{z}}}{Q-Q_{\mathrm{p}}} \mathbf{Q} \cdot \hat{\mathbf{n}}_{\mathrm{s}} \mathcal{P}_{\mathbf{s}} \mathrm{e}^{\mathrm{i} \mathbf{Q} \cdot \mathbf{R}-Q z} .
$$

We consider the edge profile to be smooth enough as to apply a local relation $\mathcal{P}_{\mathbf{s}}=C \hat{\mathbf{n}}_{\mathbf{s}}\left(\hat{\mathbf{n}}_{\mathbf{s}} \cdot \mathbf{E}^{\text {ext }}\right)$ between the dipole density $\mathcal{P}_{\mathrm{s}}$ and the external light field $\mathbf{E}^{\text {ext }}$, where $C$ is a constant coefficient. We use the value $C=1 / \pi Q_{\mathrm{p}}^{2}$, adapted from the analytical solution of the edge configuration using the Wiener-Hopf method. ${ }^{49}$ At this point, we perform the $\mathbf{Q}$ integral using complex contour methods, which result in a residue arising from the pole at $Q=Q_{\mathrm{p}}$, leading to an expression in terms of Hankel functions $H_{n}^{(1)} \equiv H_{n}^{(1)}\left(Q_{\mathrm{p}}|\mathbf{R}-\mathbf{s}|\right)$. Defining $\mathbf{t}=\mathbf{R}-\mathbf{s}$ and $\phi_{t}$ as the angle between $\mathbf{t}$ and $\hat{\mathbf{x}}$, we find

$$
\begin{aligned}
{\left[\begin{array}{c}
E_{x}^{(1)} \\
E_{y}^{(1)}
\end{array}\right]=} & \frac{\mathrm{i} Q_{\mathrm{p}} E^{\mathrm{ext}}}{2} \oint d \mathbf{s} n_{x}\left(H_{0}^{(1)}\left[\begin{array}{l}
n_{x} \\
n_{y}
\end{array}\right]\right. \\
& \left.-H_{2}^{(1)} \cos 2 \phi_{t}\left[\begin{array}{c}
n_{x} \\
-n_{y}
\end{array}\right]-H_{2}^{(1)} \sin 2 \phi_{t}\left[\begin{array}{c}
n_{y} \\
n_{x}
\end{array}\right]\right) .
\end{aligned}
$$

Finally, for the semicircular edge geometry and light polarization depicted in Fig. 1(a), Eq. (2) reduces to

$$
\mathbf{E}^{(1)}(\mathbf{R})=\frac{\mathrm{i} Q_{\mathrm{p}} a E^{\mathrm{ext}}}{2} \int_{-\pi / 2}^{\pi / 2} d \varphi \cos \varphi\left[H_{0}^{(1)} \hat{\mathbf{n}}_{\mathrm{s}}-H_{2}^{(1)} \hat{\mathbf{n}}_{\mathrm{s}}^{\prime}\right],
$$

where $\hat{\mathbf{n}}_{\mathbf{s}}$ and $\hat{\mathbf{n}}_{\mathrm{s}}^{\prime}$ are the unit vectors that form angles $\varphi$ and $2 \tan ^{-1}(y / x)-\varphi$ with respect to the $x$ axis.

Figure 1(c) shows the linear in-plane field amplitude obtained from Eq. (3) for a semicircle of radius $a=5 \lambda_{\mathrm{p}}$, assuming a plasmon propagation distance (i.e., an intensity decay by a factor $1 / \mathrm{e}$ ) given by $L_{\mathrm{p}}=10 \lambda_{\mathrm{p}}$ (note that $L_{\mathrm{p}}=1 /$ $\left.2 \operatorname{Im}\left\{Q_{\mathrm{p}}\right\}\right)$. As expected, the plasmon focal spot has a size of the order of $\lambda_{\mathrm{p}}$. We stress that the enhancement only depends on the lens geometry, the plasmon wavelength $\lambda_{\mathrm{p}}$, and the propagation distance $L_{\mathrm{p}}$, thus rendering a universal characterization that is independent of other material parameters. (a)

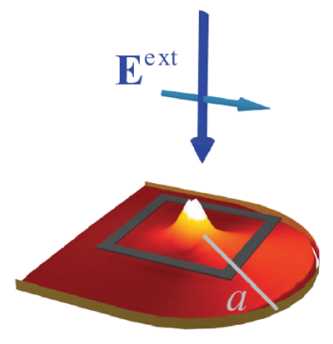

(b)

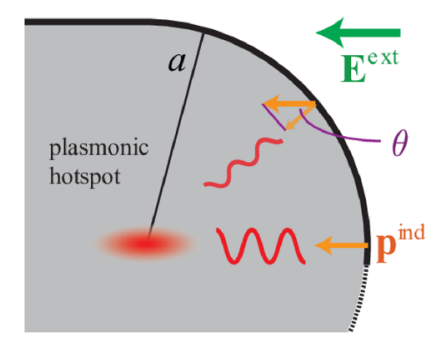

(c)

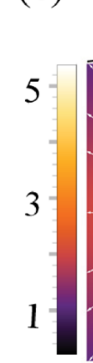

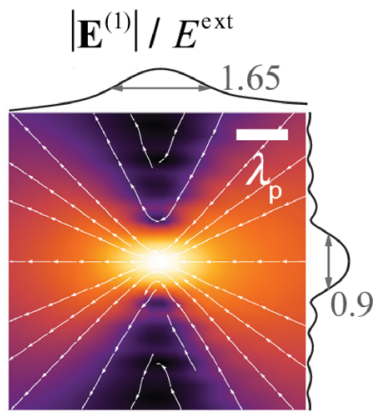

FIG. 1. Nanofocusing of graphene plasmons. (a) We consider a graphene film limited by a semicircular edge profile (radius $a \gg$ plasmon wavelength $\lambda_{\mathrm{p}}$ ) illuminated under normal incidence by a plane wave (external field $\mathbf{E}^{\text {ext }}$ ). (b) The semicircular edge couples light into plasmons, which are then focused at the center of the circle. (c) The resulting plasmon focal spot has a size comparable to $\lambda_{\mathrm{p}}$, as shown by the plasmon amplitude and instantaneous field lines, plotted here within the square region indicated in (a). The density plots are calculated for $a=5 \lambda_{\mathrm{p}}$ over a square region of side $5 \lambda_{\mathrm{p}}$. Intensity profiles across the focal spot are shown as side curves in (c), with FWHMs indicated in units of $\lambda_{\mathrm{p}}$. We assume a plasmon propagation length (1/e intensity decay) of $10 \lambda_{\mathrm{p}}$. 
We now calculate third-harmonic generation (THG) in the semicircular structure. The THG current oscillates with frequency $3 \omega$ and a local amplitude given by $\mathbf{j}_{\mathrm{NL}}^{(3)}(\mathbf{R})$ $=\sigma^{(3)} \mathbf{E}^{(1)}\left(\mathbf{E}^{(1)} \cdot \mathbf{E}^{(1)}\right)$, where for graphene doped to a Fermi energy $E_{\mathrm{F}}$, we use $\sigma^{(3)}=\left(3 \mathrm{i} e^{4} v_{\mathrm{F}}^{2} / 4 \pi \hbar^{2} E_{\mathrm{F}}\right) \times[(3 \omega+\mathrm{i} \gamma)(2 \omega$ $+\mathrm{i} \gamma)(\omega+\mathrm{i} \gamma)]^{-1}$ to model the nonlinear conductivity in the Drude model,${ }^{50}$ with a damping rate $\gamma$ related to the ratio of the propagation distance to the plasmon wavelength through $L_{\mathrm{p}} / \lambda_{\mathrm{p}}=\omega / 4 \pi \gamma$. Figure 2(b) shows the resulting THG current, exhibiting a focal spot with a size roughly reduced by a factor $\sim 1 / \sqrt{3}$ relative to the spot obtained for the linear field [cf. Figs. 1(c) and 2(b)], as expected from the reduction in size obtained when a roughly Gaussian linear profile (the linear field intensity spot) is raised to the third power (i.e., an approximate way of estimating the THG spot profile). The resulting THG emission [Fig. 2(a)] shows a distribution peaked near the surface normal direction, with more weight toward the region opposite to the curved profile because it is mainly contributed by plasmon components propagating in that direction.

We present in Fig. 3 an analysis of the performance of the focusing structure as a function of the parameters $a$ and $L_{\mathrm{p}}$. The linear field at the focal spot [Fig. 3(a)] admits from Eq. (3) the analytical expression $\mathbf{E}^{(1)}(0)=\left(E^{\text {ext }} / 2\right) \mathrm{e}^{\mathrm{i} \pi / 4}$ $\sqrt{2 \pi Q_{\mathrm{p}} a} \mathrm{e}^{\mathrm{i} Q_{\mathrm{p}} a} \hat{\mathbf{x}}$, so that noticing that $\lambda_{\mathrm{p}}=2 \pi / \operatorname{Re}\left\{Q_{\mathrm{p}}\right\}$ $\approx 2 \pi /\left|Q_{\mathrm{p}}\right|$, the enhancement of the field intensity becomes

$$
\left|\frac{\mathbf{E}^{(1)}(0)}{E^{\mathrm{ext}}}\right|^{2} \approx \frac{\pi^{2} a}{\lambda_{\mathrm{p}}} \mathrm{e}^{-a / L_{\mathrm{p}}}
$$

which is rigorously found to reach an optimum enhancement value for a radius $a=L_{\mathrm{p}}$, encompassing a compromise between field accumulation at the focus and attenuation due to propagation from the edge. For an attainable propagation distance $L_{\mathrm{p}}=15 \lambda_{\mathrm{p}}{ }^{24}$ the in-plane intensity enhancement can reach a factor of $\sim 50$, which is remarkable considering that this is the field acting inside the material. Incidentally, when considering a full circle instead of the semicircle-terminated ribbon, the field amplitude at the center is exactly zero because of symmetry (opposite sign contributions from each of the two semicircles). Notice that the level of plasmonic enhancement obtained with the semicircle-ended ribbon is uncommon inside traditional plasmonic materials, such as noble metals: the high level of optical enhancement observed
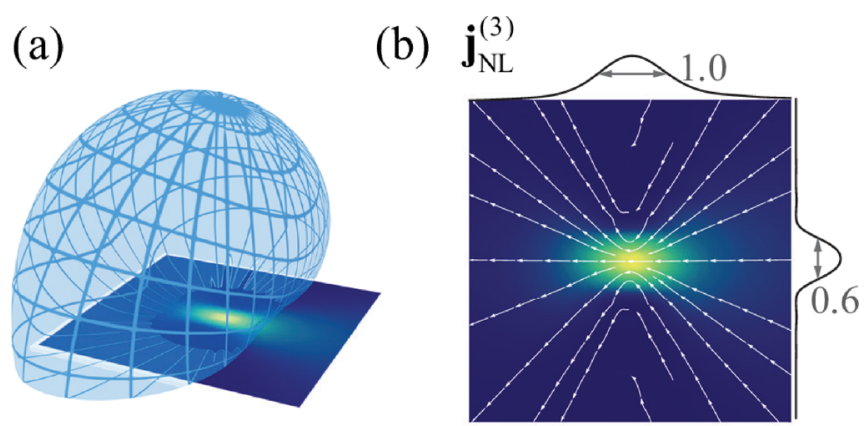

FIG. 2. Third harmonic generation (THG) through plasmon nanofocusing. We show the far-field angular distribution (a) and the instantaneous nonlinear surface current lines (b) associated with THG under the same conditions as in Fig. 1.
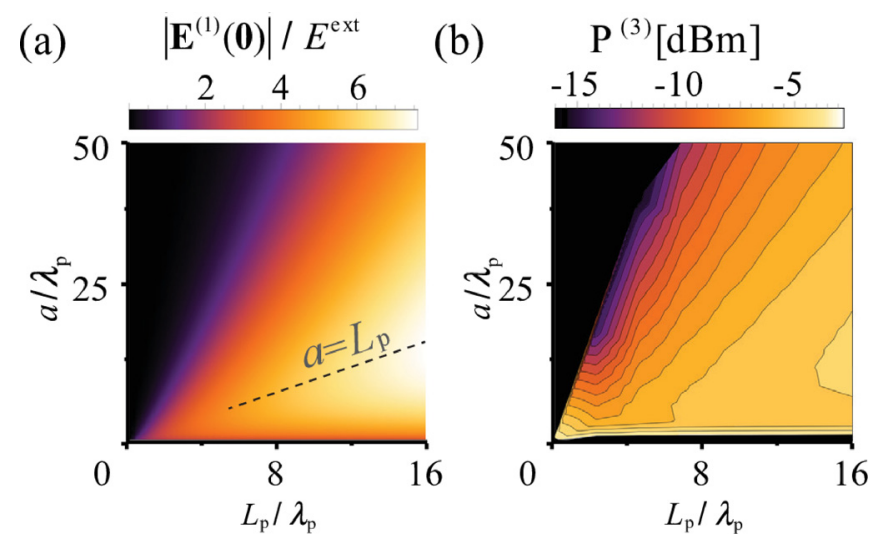

FIG. 3. Linear in-plane field enhancement and third-harmonic emission power. (a) Linear field enhancement at the center of the semicircular structure of Fig. 1(a) as a function of radius $a$ and plasmon propagation distance $L_{\mathrm{p}}$. The dashed curve shows the analytical result $a=L_{\mathrm{p}}$ for maximum enhancement as a function of $L_{\mathrm{p}}$. (b) Power of third-harmonic emission from graphene doped to a Fermi energy $E_{\mathrm{F}}=0.4 \mathrm{eV}$ for an incident intensity of $10^{13} \mathrm{~W} / \mathrm{m}^{2}$ and a plasmon energy $E_{\mathrm{p}}=0.2 \mathrm{eV}$, corresponding to $\lambda_{\mathrm{p}}=181 \mathrm{~nm}$ (see the main text for details on the nonlinear conductivity).

in noble metal nanoparticles is associated with electric field components oriented along the local surface normal direction; these components can reach dramatically strong enhancement for resonant morphologies (e.g., high-aspect ratios or narrow inter-metallic gaps), generally involving large values of the permittivity-losses in these materials are too high otherwise - and so, the field inside the nanoparticle is severely reduced to levels similar to those of the incident light in virtue of the continuity of the electric displacement. Finally, we show in Fig. 3(b) the angle-integrated THG, which is observed to roughly follow a trend as expected from the sixth power of the linear field amplitude. Incidentally, the overall conversion efficiency, which we can define as the emitted power divided by the power incident on a semicircle, takes a value of $\sim 10^{-6}$.

The light angle of incidence could be exploited to control the position of the plasmon focal spot, and for more complex edge profiles, light polarization may provide an additional degree of freedom. To illustrate this concept, we consider the shape of a half-ribbon lens capable of focusing p-polarized light incident in the $x-z$ plane with an angle $\theta$ relative to the surface-normal direction $z$. Taking the focus at the origin $x=y=0$, the phase-preserving ribbon-end edge profile is elliptical and given by $\alpha_{-}(x-b)^{2}+y^{2}=a^{2} \alpha_{+}$, where $\alpha_{ \pm}=1 \pm l^{2} \sin ^{2} \theta, b=\left(a l / \alpha_{-}\right) \sin \theta$, and $l=\lambda_{\mathrm{p}} / \lambda_{0}$. For highly confined plasmons $(l \ll 1)$, this produces a nearly circular profile although the center of the structure $(x=b)$ can be displaced by a sizable fraction of $\lambda_{\mathrm{p}}$, as shown in Fig. 4(a). The field enhancement and resulting THG are also nearly indistinguishable in the semicircle and the phasepreserving ellipse, and although they decrease with $\theta$, their values stay high up to incidence directions for which the focus displacement is $\sim \lambda_{\mathrm{p}}$ (Fig. 4).

The proposed approach toward plasmon focusing can be extended to any polariton-supporting surface, including thin metal films, under the condition that they exhibit long propagation distances $L_{\mathrm{p}}$ and short wavelengths $\lambda_{\mathrm{p}}$, reaching a maximum focal intensity enhancement $\pi^{2} L_{\mathrm{p}} / \mathrm{e} \lambda_{\mathrm{p}} \approx 3.6 L_{\mathrm{p}} / \lambda_{\mathrm{p}}$ [see Eq. (4)]. We envision further optimization of the edge 
(a)

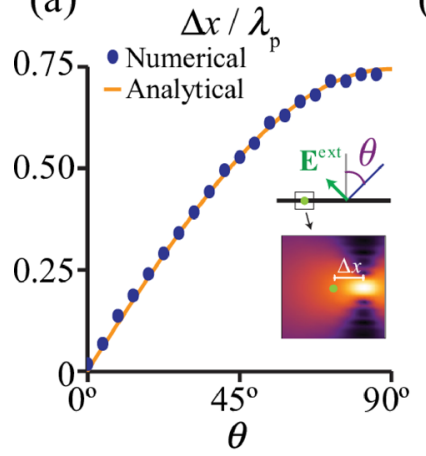

(b)

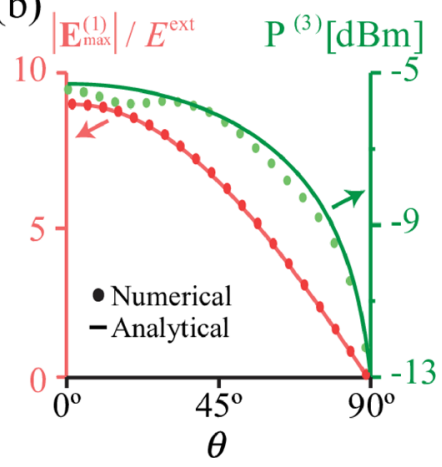

FIG. 4. Control of the plasmon focal spot through the incidence angle. (a) Displacement of the spot center in a semicircular lens as a function of incidence angle $\theta$ relative to the surface normal for $a=L_{\mathrm{p}}=25 \lambda_{\mathrm{p}}$. The inset shows a near-field linear plot for $\theta=45^{\circ}$. (b) Linear field enhancement (left scale) and THG power (right scale) under the conditions of (a) for $E_{\mathrm{F}}$ $=0.4 \mathrm{eV}, E_{\mathrm{p}}=0.2 \mathrm{eV}$, and $10^{13} \mathrm{~W} / \mathrm{m}^{2}$ incidence intensity. The results obtained through numerical evaluation of the field using Eq. (3) (symbols) are compared with closed-form analytical expressions (curves). The analytical THG is approximated as the third power of the field intensity.

profile to tailor the spatial distribution of the focal spot. Besides harmonic generation, either in the polariton-supporting materials or in the surrounding media, this concept could find application in sensing, for example, by revealing the presence of analytes in the focal region through their optical scattering signatures. Edge scattering can also be used in reversed geometry to out-couple plasmons generated at the focal spot, thus producing a propagating light signal. From a more general perspective, engineered edge profiles can serve as interconnects between different focal spots (e.g., the foci of a graphene ellipse).

This work was supported in part by the Spanish MINECO (MAT2017-88492-R and SEV2015-0522), the European Commission (Graphene Flagship 696656 and FP7ICT-2013-613024-GRASP), the Catalan CERCA Program, Fundació Privada Cellex, and AGAUR (Grant Nos. FI_B 00492-2015 and 2014 SGR 1400).

${ }^{1}$ M. Danckwerts and L. Novotny, Phys. Rev. Lett. 98, 026104 (2007).

${ }^{2}$ A. R. Davoyan, I. V. Shadrivov, and Y. S. Kivshar, Opt. Express 16, 21209 (2008).

${ }^{3}$ S. Palomba and L. Novotny, Phys. Rev. Lett. 101, 056802 (2008).

${ }^{4}$ A. K. Singh, D. Senapati, A. Neely, G. Kolawole, C. Hawker, and P. C. Ray, Chem. Phys. Lett. 481, 94 (2009).

${ }^{5}$ Y. Pu, R. Grange, C.-L. Hsieh, and D. Psaltis, Phys. Rev. Lett. 104, 207402 (2010)

${ }^{6}$ J. A. Schuller, E. S. Barnard, W. Cai, Y. C. Jun, J. S. White, and M. L. Brongersma, Nat. Mater. 9, 193 (2010).

${ }^{7}$ P.-Y. Chen, C. Argyropoulos, and A. Alù, Nanophotonics 1, 221 (2012).

${ }^{8}$ M. Kauranen and A. V. Zayats, Nat. Photonics 6, 737 (2012).

${ }^{9}$ M. Mesch, B. Metzger, M. Hentschel, and H. Giessen, Nano Lett. 16, 3155 (2016).

${ }^{10}$ R. A. Álvarez-Puebla, L. M. Liz-Marzán, and F. J. García de Abajo, J. Phys. Chem. Lett. 1, 2428 (2010).

${ }^{11}$ M. M. Jadidi, J. C. Köonig-Otto, S. Winnerl, A. B. Sushkov, H. D. Drew, T. E. Murphy, and M. Mittendorff, Nano Lett. 16, 2734 (2016).

${ }^{12}$ A. G. Curto and F. J. García de Abajo, Opt. Express 17, 17801 (2009).

${ }^{13}$ K. R. Li, M. I. Stockman, and D. J. Bergman, Phys. Rev. Lett. 91, 227402 (2003).

${ }^{14}$ M. I. Stockman, Phys. Rev. Lett. 93, 137404 (2004).
${ }^{15}$ O. Lozan, R. Sundararaman, B. Ea-Kim, J.-M. Rampnoux, P. Narang, S. Dilhaire, and P. Lalanne, Nat. Commun. 8, 1656 (2017).

${ }^{16}$ C. Ropers, D. R. Solli, C. P. Schulz, C. Lienau, and T. Elsaesser, Phys. Rev. Lett. 98, 043907 (2007).

${ }^{17}$ Z. Fang, Q. Peng, W. Song, F. Hao, J. Wang, P. Nordlander, and X. Zhu, Nano Lett. 11, 893 (2011).

${ }^{18}$ L. Du and D. Tang, J. Opt. Soc. Am. A 31, 691 (2014).

${ }^{19}$ Z. Fei, G. O. Andreev, W. Bao, L. M. Zhang, A. S. McLeod, C. Wang, M. K. Stewart, Z. Zhao, G. Dominguez, M. Thiemens et al., Nano Lett. 11, 4701 (2011).

${ }^{20}$ Z. Fei, A. S. Rodin, G. O. Andreev, W. Bao, A. S. McLeod, M. Wagner, L. M. Zhang, Z. Zhao, M. Thiemens, G. Dominguez et al., Nature 487, 82 (2012).

${ }^{21}$ J. Chen, M. Badioli, P. Alonso-González, S. Thongrattanasiri, F. Huth, J. Osmond, M. Spasenović, A. Centeno, A. Pesquera, P. Godignon et al., Nature 487, 77 (2012).

${ }^{22}$ H. Yan, T. Low, W. Zhu, Y. Wu, M. Freitag, X. Li, F. Guinea, P. Avouris, and F. Xia, Nat. Photonics 7, 394 (2013).

${ }^{23}$ F. J. García de Abajo, ACS Photonics 1, 135 (2014).

${ }^{24}$ A. Woessner, M. B. Lundeberg, Y. Gao, A. Principi, P. Alonso-González, M. Carrega, K. Watanabe, T. Taniguchi, G. Vignale, M. Polini et al., Nat. Mater. 14, 421 (2015).

${ }^{25}$ T. Low, R. Roldán, H. Wang, F. Xia, P. Avouris, L. Martín Moreno, and F. Guinea, Phys. Rev. Lett. 113, 106802 (2014).

${ }^{26}$ M. A. Huber, F. Mooshammer, M. Plankl, L. Viti, F. Sandner, L. Z. Kastner, T. Frank, J. Fabian, M. S. Vitiello, T. L. Cocker et al., Nat. Nanotechnol. 12, 207 (2017).

${ }^{27}$ F. Moresco, M. Rocca, T. Hildebrandt, and M. Henzler, Phys. Rev. Lett. 83, 2238 (1999).

${ }^{28}$ D. N. Basov, M. M. Fogler, and F. J. García de Abajo, Science 354, aag1992 (2016).

${ }^{29}$ R. C. McPhedran, G. H. Derrick, and L. C. Botten, in Electromagnetic Theory of Gratings, edited by R. Petit (Springer-Verlag, Berlin, 1980), pp. 227-276.

${ }^{30}$ B. Zhao and Z. M. Zhang, ACS Photonics 2, 1611 (2015).

${ }^{31}$ L. Novotny and B. Hecht, Principles of Nano-Optics (Cambridge University Press, New York, 2006).

${ }^{32}$ G. V. Pavan Kumar, J. Nanophotonics 6, 064503 (2012).

${ }^{33}$ C. T. Ertsgaard, R. M. McKoskey, I. S. Rich, and N. C. Lindquist, ACS Nano 8, 10941 (2014).

${ }^{34}$ Y.-J. Oh, M. Kang, and M. P. K.-H. Jeong, BioChip J. 10, 297 (2016).

${ }^{35}$ D. M. Solís, J. M. Taboada, F. Obelleiro, L. M. Liz-Marzán, and F. J. García de Abajo, ACS Photonics 4, 329 (2017).

${ }^{36}$ A. Hartstein, J. R. Kirtley, and J. C. Tsang, Phys. Rev. Lett. 45, 201 (1980).

${ }^{37}$ J. Chae, B. Lahiri, and A. Centrone, ACS Photonics 3, 87 (2016).

${ }^{38}$ E. Hendry, P. J. Hale, J. Moger, A. K. Savchenko, and S. A. Mikhailov, Phys. Rev. Lett. 105, 097401 (2010).

${ }^{39}$ R. Wu, Y. Zhang, S. Yan, F. Bian, W. Wang, X. Bai, X. Lu, J. Zhao, and E. Wang, Nano Lett. 11, 5159 (2011).

${ }^{40}$ S. A. Mikhailov and K. Ziegler, J. Phys. Condens. Matter 20, 384204 (2008).

${ }^{41}$ S. A. Mikhailov, "Carbon nanotubes and graphene for photonic applications," in Electromagnetic Nonlinearities in Graphene (Woodhead Publishing Limited, Cambridge, 2013), p. 171.

${ }^{42}$ J. D. Cox and F. J. García de Abajo, Nat. Commun. 5, 5725 (2014).

${ }^{43}$ T. J. Constant, S. M. Hornett, D. E. Chang, and E. Hendry, Nat. Phys. 12, 124 (2016).

${ }^{44}$ T. Christensen, W. Yan, A.-P. Jauho, M. Wubs, and N. A. Mortensen, Phys. Rev. B 92, 121407(R) (2015).

${ }^{45}$ J. L. Cheng, N. Vermeulen, and J. E. Sipe, Phys. Rev. B 91, 235320 (2015).

${ }^{46}$ S. A. Mikhailov, Phys. Rev. B 93, 085403 (2016).

${ }^{47}$ J. D. Cox, R. Yu, and F. J. García de Abajo, Phys. Rev. B 96, 045442 (2017).

${ }^{48}$ F. J. García de Abajo, ACS Nano 7, 11409 (2013).

${ }^{49}$ Z. Lei, F. Xiu-Li, and Y. Jun-Zhong, Commun. Theor. Phys. 61, 751 (2014).

${ }^{50}$ N. M. R. Peres, Y. V. Bludov, J. E. Santos, A.-P. Jauho, and M. I. Vasilevskiy, Phys. Rev. B 90, 125425 (2014). 